\title{
The association between wearable activity monitor metrics and performance status in oncology: a systematic review
}

\author{
Milan $\operatorname{Kos}^{1} \mathbb{D}$ - Esther N. Pijnappel ${ }^{1} \cdot$ Laurien M. Buffart ${ }^{2}$ - Britt R. Balvers ${ }^{1} \cdot$ Caroline S. Kampshoff $^{3,4}$. \\ Johanna W. Wilmink ${ }^{1} \cdot$ Hanneke W. M. van Laarhoven ${ }^{1,3} \cdot$ Martijn G. H. van Oijen $^{1}$
}

Received: 16 November 2020 / Accepted: 19 April 2021 / Published online: 12 June 2021

(c) The Author(s) 2021

\begin{abstract}
Purpose The expanding armamentarium of wearable activity monitors (WAMs) offers new opportunities to supplement physician-assessed performance status (PS) with real-life patient activity data. These data could guide clinical decision making or serve as a measure of treatment outcome. However, information on the association between physical activity (PA) and sedentary behavior (SB) monitored with wearables (i.e., WAM metrics) and PS in patients with cancer is needed. Therefore, we conducted a systematic review to examine the association between WAM metrics and PS in patients with cancer.

Methods We searched MEDLINE and Embase for studies that assessed the association between WAM metrics and performance status among adults with cancer. We extracted information on study design and population, WAM type and different activity metrics, outcome definitions, and results. Included studies were subjected to risk of bias assessment and subsequent best evidence synthesis.

Results Fourteen studies were included in this review. All studies reported on different combinations of WAM metrics including: daily steps $(n=8), \mathrm{SB}(n=5)$, mean activity counts $(n=4)$, dichotomous circadian rest-activity index $(n=3)$, and time spent in moderate-to-vigorous PA (MVPA) $(n=3)$. Much heterogeneity was observed regarding study population, WAM used, and reporting of results. We found moderate evidence for a positive weak-to-moderate association between WAM-assessed PA and PS and a weak-to-moderate negative association between WAM-assessed SB metrics and PS.

Conclusion Weak-to-moderate associations between WAM metrics and PS suggest that WAM data and physician-assessed PS cannot be used interchangeably. Instead, WAM data could serve as a dynamic and objective supplement measurement of patients' physical performance.
\end{abstract}

Keywords Cancer $\cdot$ Performance status $\cdot$ Physical activity $\cdot$ Physical function $\cdot$ Sedentary behavior $\cdot$ Wearable activity monitor

Milan Kos

milan.kos@amsterdamumc.nl

1 Department of Medical Oncology, Cancer Center Amsterdam, Amsterdam UMC, University of Amsterdam, Meibergdreef 9, Amsterdam, The Netherlands

2 Department of Physiology, Radboud Institute for Health Sciences, Radboud University Medical Center, Philips van Leydenlaan 15, Nijmegen, The Netherlands

3 Department of Medical Oncology, Cancer Center Amsterdam, Amsterdam UMC, Vrije Universiteit Amsterdam, De Boelelaan 1117, Amsterdam, The Netherlands

4 Center for Quality of Life, The Netherlands Cancer Institute, Amsterdam, The Netherlands

\section{Introduction}

Patients' performance status is a significant prognostic and predictive factor for clinically relevant outcomes, such as progression-free and overall survival of patients with cancer [1-3]. It therefore is one of the key inclusion criteria for clinical trials and often serves as stratification factor in trial design and analyses. Moreover, in daily clinical practice it is used to decide whether a patient is fit for systemic therapy [4], or eligible for early phase clinical trials. Patients' performance status is determined by healthcare professionals using either the Karnofsky Performance Status (KPS) or the Eastern Cooperative Oncology Group Performance Status (ECOG-PS, also known as WHO PS) [5, 6]. Both methods have proven their clinical relevance over the past decades 
and are widely used. However, these methods also present with potential bias and limitations [7]. First, performance status scoring depends on the oncologists subjective rating of a patient's health and functioning with no standardized process for this assessment, making it prone to under- and overestimation, and inter-observer differences [8-12]. Second, performance status assessment may be susceptible to response and recall bias as it relies on patient-reported physical activity and functioning [13]. Third, both KPS and ECOG-PS are static measurements that are only captured during scheduled visits, whereas patient's physical performance is a dynamic process that may change on a daily basis during the course of treatment. As a result, recent reviews have accentuated the need for a tool that can assess patient's physical performance objectively in a more dynamic fashion $[7,14]$.

The expanding armamentarium of wearable activity monitors (e.g., accelerometers, pedometers, fitness trackers, smartwatches, and smartphones) offers new opportunities to supplement physician-assessed performance status with objective assessments of physical activity and sedentary behavior, which are passively gathered in a non-obtrusive manner. It is even hypothesized that wearable activity monitor metrics might prove superior to clinician-rated performance status or patient-reported data in terms of accurately discriminating between the heterogeneous spectrum of cancer patients $[7,14]$. Therefore, wearable activity monitorderived data may assist healthcare professionals in making treatment decisions (e.g., mono vs doublet vs triplet chemotherapy) for individual patients $[15,16]$ and could be useful in assuring that performance status of patients enrolled in clinical trials is recorded accurately [7]. Multiple recent clinical studies have demonstrated the feasibility of using wearable activity monitors to assess physical activity and sedentary behavior in patients with cancer. However, no aggregated evidence is available about the use of wearable activity monitor-derived physical activity and sedentary behavior metrics to supplement physician-assessed performance status. As a first step towards this purpose, we conducted a systematic review on the association between wearable activity monitor physical activity and sedentary behavior metrics and performance status in patients with cancer.

\section{Methods}

A systematic review of available literature was conducted in agreement with the guideline for preferred reporting items for systematic reviews and meta-analyses (PRISMA statement) [17]. This review has been registered in PROSPERO (CRD4202013865).

\section{Literature search}

MEDLINE® and Embase databases were searched from inception until April 2020 to identify all relevant published articles. An experienced clinical librarian from the Amsterdam UMC was consulted for the development of the search strategy. Relevant keywords included terms related to wearable activity monitor metrics AND cancer population AND wearable activity monitors (e.g., fitness trackers, smartwatches, accelerometers, pedometers, actigraphs, and inclinometers). The complete search strategies are presented in Supplementary Table 1. Moreover, crossreferencing was performed to identify additional relevant studies for the systematic review.

\section{Eligibility criteria}

Studies were included if they (1) were conducted among adults ( $\geq 18$ years) with cancer, (2) objectively measured physical activity or sedentary behavior using wearable activity monitors in the outpatient setting, (3) measured physician-assessed performance status, (4) quantitatively assessed an association between wearable activity monitor metrics and clinician-assessed performance status, and (5) had a full text available in English.

\section{Definitions of wearable activity monitor metrics for physical activity, sedentary behavior, and circadian rest-activity rhythm}

Many different activity-related wearable activity monitor metrics are being used in research and reported wearable activity monitor metrics often depend on the used device. Four main categories of wearable activity monitor metrics relevant for this review can be identified: (1) accelerometer-related activity count-based metrics that capture the duration and intensity of accelerations in counts per minute and/or hours. Moreover, these intensities can be used to determine absolute or relative time spent in sedentary behavior, light physical activity (LPA), or moderate-tovigorous physical activity (MVPA) based on predefined cut-points. (2) Posture-based measures that define hours of percentage of time per day spent sitting/lying (i.e., sedentary), standing, or stepping. (3) Steps-based measurement that estimate the number of steps per day using an algorithm that determines whether accelerometer measurements meet the threshold to be counted as a step. (4) Circadian rhythm rest-activity actigraphy parameters like, the dichotomous in-bed vs out-of-bed index $(\mathrm{I}<\mathrm{O})$ and mean activity level (MeanAct). 


\section{Selection process and eligibility criteria}

Titles and abstracts of articles identified by the electronic database searches were extracted and duplicates were removed. Two reviewers (MK and EP or BB) independently screened the records of the initial search to select potentially relevant articles that were subsequently subjected to full-text screening for eligibility. Any discrepancies between reviewers were discussed in person. If no agreement was reached, discrepancies were referred to a third reviewer $(\mathrm{MvO})$ before a final decision was made on inclusion.

\section{Data extraction}

Data on study design and population, physical activity/sedentary behavior measurement characteristics and protocols, wearable activity monitors, and outcome measures were extracted using a standardized form including the following items: first author, year of publication, country, study design, number of included patients, type of cancer and disease stage, current treatment, comorbidities, performance status scale used, measure of physical activity or sedentary behavior (including definitions and cutoff points), devices used for physical activity/sedentary behavior measurements, wear location of devices, statistical methods and analyses, and results on association between wearable activity monitor metrics and performance status. If point estimates (e.g., mean, median) were only depicted in figures, authors were first contacted and asked to provide these point estimates. If we did not receive data from the authors, we used open source software WebPlotDigitizer (version 4.3) to estimate the point estimates and corresponding measures for variability (e.g., IQR or SD) from the figures [18-20]. The widely used empirical classifications proposed by Evans were used for interpreting correlation strengths [21]. Correlation coefficients of $0-0.19$ were interpreted as very weak, $0.2-0.39$ as weak, $0.4-0.59$ as moderate, $0.6-0.79$ as strong, and $0.8-1$ as very strong.

\section{Risk of bias assessment}

The risk of bias of included studies was scored independently by two reviewers (MK and EP) using a risk of bias assessment tool based on the guideline for assessing quality in prognostic studies [22]. These guidelines comprise 6 potential biases (i.e., study participation, study attrition, prognostic factor measurement, outcome measurement, confounding measurement and account, and analysis) and were applied on the basis of relevance to this systematic review. Subsequently, the potential biases were translated into a 15-point quality criteria list, based on previously published risk of bias criteria lists (Supplementary Table 2) [23-26]. Furthermore, quality items were categorized as informative (I, 5 items) or validity/precision (V/P, 10 items) [23, 25]. If the study provided adequate information and met the criterion of the quality item, one single point was appointed. If the study provided insufficient information or did not meet the criterion of the quality item, no point was appointed. If the study referred to another article for relevant information regarding the quality item, that article was reviewed to score the item. Disagreements regarding the risk of bias assessment were resolved by discussion and, if no agreement could be reached, by consulting the third reviewer $(\mathrm{MvO})$. A total risk of bias score was calculated for each included study by dividing the amount of positively scored validity/precision items by the total amount of validity/precision items (i.e., $10)$, resulting in a score between 0 and 1 , presented as a percentage. The five informative items were not included in the final calculation, as these items represent descriptive information only [23, 25]. In line with previous reviews [23, 27], a study with a score $\geq 70 \%$ was considered to be of "low risk of bias," whereas as a study with a score $<70 \%$ was considered to be of "high risk of bias" [23, 27].

\section{Level of evidence}

A 3-level scoring system for best evidence synthesis based on the number, methodological quality, and consistency of outcomes of the different studies was applied to synthesize the methodological quality of the included studies [23, 27, 28]: (1) strong evidence; provided by general consistent findings in multiple ( $\geq 2)$ studies with low risk of bias, (2) moderate evidence; provided by general consistent findings in 1 study with low risk of bias and 1 or more studies of high risk of bias or general consistent findings in multiple $(\geq 2)$ studies with high risk of bias, and (3) insufficient evidence; only one study available or inconsistent findings in multiple $(\geq 2)$ studies. Results were considered general consistent when at least $75 \%$ of studies showed results in the same direction.

\section{Results}

The combined literature search yielded 1511 unique records. The result of the systematic literature search and subsequent selection of studies is depicted in Fig. 1. After initial screening, 373 articles were retrieved in full-text and checked for eligibility. Cross-referencing provided one additional study [29] that could be included in the systematic review. Ultimately, 14 studies met the eligibility criteria and where included for further analysis. Table 1 provides an overview of the baseline characteristics of the included articles.

\section{Risk of bias assessment}

Results of the risk of bias assessment are depicted in Table 2. The median methodological quality of the included 
Fig. 1 Flowchart of literature search and inclusion of studies. PA, physical activity; SB, sedentary behavior; KPS, Karnofsky Performance Status; ECOG-PS, Eastern Conference Oncology Group Performance Status; CircAct, circadian rest-activity rhythm; PS, performance status; $n$, number of studies

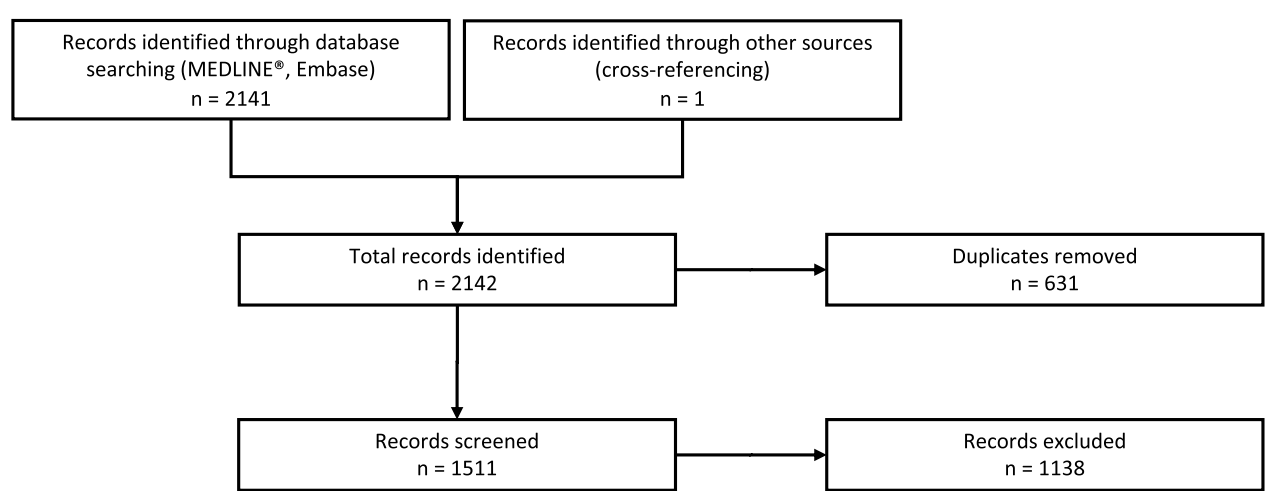

Full-text articles excluded: $n=359$

- Conference abstract ( $n=1)$

- No adult cancer population $(n=8)$

- Bone or soft-tissue malignancies $(n=6)$

- No PA, SB, or CircAct reported as outcome $(n=30)$

Full-text articles assessed for eligibility

PA or SB as outcome not measured with wearable activity monitor $(n=19)$

- KPS or ECOG-PS not measured in study ( $n=250)$

- Only patient-assessed PS $(n=2)$

- No described association between

PA/SB/CircAct and PS in study $(n=42)$

- Inpatient setting $(n=1)$ studies was $40 \%$ and ranged from 20 to $60 \%$. Only $29 \%$ of included studies had an adequate participation rate $(>80 \%)$. Most $(64 \%)$ studies had a small sample size $(n<100)$. None of the studies adequately described methods used for dealing with missing physical activity data, and the majority of studies (79\%) used a combination of device and wear-time protocol that has not been adequately validated in the studied population.

\section{Wearable activity monitors and physical activity/ sedentary behavior metrics}

The characteristics of the used wearable activity monitors and physical activity/sedentary behavior metrics are summarized in Table 3. A total of 9 different devices were used in the included articles and worn on the wrist ( 9 studies [29-37]), thigh (3 studies [38-40]), hip (1 study [41]), or waist (1 study [42]). Furthermore, different wear-time protocols were used between studies. Included articles reported on a total of 12 different wearable activity monitor metrics: 8 studies reported on steps taken [33-35, 37-41], 6 on sedentary behavior (i.e., posture-based and activity counts-based) [34, 36, 38, 39, 41, 42], 4 on mean daily activity counts [29-32], 3 on time spent in MVPA [40-42], 3 on the dichotomy in-bed versus out-of-bed index $\mathrm{I}<\mathrm{O}$ [30-32], and 2 on time spent in light physical activity (LPA) [41, 42]. Other reported PA metrics included: time spent stepping [38-40], time spent standing [29, 31], distance walked [33], total metabolic expenditure per day [38], and daily floors climbed [33] (Table 3). Different methods, definitions, and cut-points were used for sedentary behavior, LPA, and MVPA based on the devices used.

\section{Physical activity and sedentary behavior outcomes per ECOG-PS group}

Table 4 provides an overview of the included studies that reported on the physical activity and sedentary behavior outcomes per ECOG-PS group. All studies that reported on mean daily steps per ECOG-PS group found significant between-group differences with more daily steps in patients with better performance status. Moreover, two studies [38, 39] reported that patients with better performance status spent significantly less time sitting/lying (i.e., sedentary) and significantly more time standing and stepping as compared to patients with worse performance status. Three studies reporting on mean total activity counts per day and the circadian rest-activity dichotomous index $\mathrm{I}<\mathrm{O}$, all showed significantly more activity counts and per day and less circadian rhythm disruption in patients with better performance status [30-32]. Regarding intensity-based wearable activity monitor metrics, one study reported that patients with good performance status spent significantly more time in LPA and MVPA and significantly less time sedentary as compared 
Table 1 Baseline characteristics of the included studies $(n=14)$

\begin{tabular}{|c|c|c|c|c|c|c|c|c|}
\hline $\begin{array}{l}\text { Study } \\
\text { first author } \\
\text { (year) }\end{array}$ & $\mathrm{n}$ & Female & $\begin{array}{l}\text { Age } \\
\text { (years) }\end{array}$ & ECOG-PS & KPS & Cancer type & Disease stage & $\begin{array}{l}\text { Treatment during } \\
\text { study }\end{array}$ \\
\hline Mormont (2000) & 192 & $64(33.3 \%)$ & $58[20-75]^{\mathrm{a}}$ & $\begin{array}{l}\text { ECOG 0: } 123 \\
(64.1 \%) \\
\text { ECOG 1: } 55 \\
(28.6 \%) \\
\text { ECOG 2: } 14 \\
(7.3 \%)\end{array}$ & & Colorectal & Stage IV & Chemotherapy \\
\hline Roscoe (2002) & 78 & $78(100 \%)$ & $52[34-79]^{\mathrm{a}}$ & & $89[70-100]^{\mathrm{a}}$ & Breast & & Chemotherapy \\
\hline $\begin{array}{l}\text { Innominato } \\
(2009)\end{array}$ & 130 & $56(43.1 \%)$ & $60[22-76]^{\mathrm{b}}$ & $\begin{array}{l}\text { ECOG 0: } 70 \\
(53.8 \%) \\
\text { ECOG 1: } 45 \\
(34.6 \%) \\
\text { ECOG 2: } 15 \\
(11.5 \%)\end{array}$ & & Colorectal & Stage IV & Chemotherapy \\
\hline Ferriolli (2012) & 53 & $13(24.5 \%)$ & $64(9)^{\mathrm{c}}$ & $\begin{array}{c}\text { ECOG 0: } 19 \\
(35.8 \%) \\
\text { ECOG 1: } 22 \\
(41.5 \%) \\
\text { ECOG 2: } 11 \\
(20.8 \%) \\
\text { ECOG 3: } 1 \\
(1.9 \%)\end{array}$ & $\begin{array}{l}85 \pm 11 \\
{[60-100]^{\mathrm{d}}}\end{array}$ & $\begin{array}{l}\text { Upper gastroin- } \\
\text { testinal }\end{array}$ & & $\begin{array}{l}\text { Surgery: } 37 \\
(69.8 \%) \\
\text { Palliative chemo- } \\
\text { therapy: } 16 \\
(30.2 \%)\end{array}$ \\
\hline $\begin{array}{l}\text { Maddocks } \\
\text { (2012) }\end{array}$ & 84 & $30(35.7 \%)$ & $66[41-86]^{\mathrm{a}}$ & $\begin{array}{l}\text { ECOG 0: } 16 \\
(19.0 \%) \\
\text { ECOG 1: } 47 \\
(56.0 \%) \\
\text { ECOG 2: } 21 \\
(25.0 \%)\end{array}$ & & $\begin{array}{l}\text { NSCLC: } 71 \\
(84.5 \%) \\
\text { SCLC: } 8(9.5 \%) \\
\text { Mesothelioma: } \\
5(6.0 \%)\end{array}$ & $\begin{array}{l}\text { Stage IIIb: } 43 \\
(51.2 \%) \\
\text { Stage IV: } 41 \\
(48.8 \%)\end{array}$ & $\begin{array}{l}\text { No treatment past } \\
4 \text { weeks and } \\
\text { scheduled for } \\
\text { palliative treat- } \\
\text { ment }\end{array}$ \\
\hline $\begin{array}{l}\text { Broderick } \\
\text { (2014) }\end{array}$ & 100 & $89(89.0 \%)$ & $54.7[24-82]^{\mathrm{a}}$ & $\begin{array}{l}\text { ECOG 0: } 28 \\
(28.0 \%) \\
\text { ECOG 1: } 61 \\
(61.0 \%) \\
\text { ECOG 2-3: } 11 \\
(11.0 \%)\end{array}$ & & $\begin{array}{l}\text { Breast: } 68 \\
(68.0 \%) \\
\text { Lung/thoracic: } \\
10(10.0 \%) \\
\text { Gynecological: } \\
10(10.0 \%) \\
\text { Other: } 12 \\
(12.0 \%)\end{array}$ & & Chemotherapy \\
\hline $\begin{array}{l}\text { Lévi (2014) } \\
\text { (cohort III) }\end{array}$ & 142 & $55(38.7 \%)$ & $60[21-83]^{\mathrm{b}}$ & $\begin{array}{c}\text { ECOG 0: } 84 \\
(59.6 \%) \\
\text { ECOG 1: } 44 \\
(31.2 \%) \\
\text { ECOG 2: } 13 \\
(9.2 \%) \\
\text { Unknown: } 1 \\
(0.7 \%)\end{array}$ & & Colorectal & Stage IV & Chemotherapy \\
\hline Jeffery (2017) & 46 & $13(28.3 \%)$ & $68.5(7.9)^{\mathrm{c}}$ & $\begin{array}{c}\text { ECOG 0: } 18 \\
(39.1 \%) \\
\text { ECOG 1: } 13 \\
(28.3 \%) \\
\text { ECOG 2: } 7 \\
(15.2 \%) \\
\text { ECOG 3: } 6 \\
(13.0 \%)\end{array}$ & & $\begin{array}{l}\text { Mesothelioma: } \\
30(65.2 \%) \\
\text { Lung cancer: } 11 \\
(23.9 \%) \\
\text { Other: } 5 \\
(10.9 \%)\end{array}$ & $\begin{array}{l}\text { Patients with } \\
\text { malignant } \\
\text { pleural effu- } \\
\text { sion }\end{array}$ & Chemotherapy \\
\hline
\end{tabular}


Table 1 (continued)

\begin{tabular}{|c|c|c|c|c|c|c|c|c|}
\hline $\begin{array}{l}\text { Study } \\
\text { first author } \\
\text { (year) }\end{array}$ & $\mathrm{n}$ & Female & $\begin{array}{l}\text { Age } \\
\text { (years) }\end{array}$ & ECOG-PS & KPS & Cancer type & Disease stage & $\begin{array}{l}\text { Treatment during } \\
\text { study }\end{array}$ \\
\hline Dennett (2018) & 49 & $33(67.3 \%)$ & $63[27-77]^{\mathrm{a}}$ & & $\begin{array}{l}80.8(10.4)^{\mathrm{c}} \\
80[60-100]^{\mathrm{a}}\end{array}$ & $\begin{array}{l}\text { Breast: } 24 \\
(49.0 \%) \\
\text { Prostate: } 5 \\
(10.2 \%) \\
\text { NHL: } 5(10.2 \%) \\
\text { Other: } 16 \\
(20.7 \%)^{*}\end{array}$ & & $\begin{array}{l}\text { No therapy: } 19 \\
(38.8 \%) \\
\text { Hormone therapy: } \\
12(24.5 \%) \\
\text { Chemotherapy: } 20 \\
(20.4 \%) \\
\text { Targeted therapy: } \\
6(12.2 \%) \\
\text { Radiotherapy: } 5 \\
(10.2 \%)^{* *}\end{array}$ \\
\hline Gresham (2018) & 37 & $17(45,9 \%)$ & $62[34-81]^{\mathrm{a}}$ & $\begin{array}{l}\text { ECOG 0: } 9 \\
(24.3 \%) \\
\text { ECOG 1: } 13 \\
(35.1 \%) \\
\text { ECOG 2: } 9 \\
(24.3 \%) \\
\text { ECOG 3: } 6 \\
(16.2 \%)\end{array}$ & $\begin{array}{c}\text { KPS 100: } 6 \\
(16.2 \%) \\
\text { KPS 90: } 5 \\
(13.5 \%) \\
\text { KPS 80: } 9 \\
(24.3 \%) \\
\text { KPS 70: } 8 \\
(21.6 \%) \\
\text { KPS 60: } 3 \\
(8.1 \%) \\
\text { KPS 50: } 5 \\
(13.5 \%) \\
\text { KPS < } 50: 1 \\
(2.7 \%)\end{array}$ & $\begin{array}{l}\text { Pancreas: } 27 \\
(73.0 \%) \\
\text { Other gastro- } \\
\text { intestinal: } 7 \\
(18.9 \%) \\
\text { Other: } 3(8.1 \%)\end{array}$ & $\begin{array}{l}\text { Stage IIIB: } 34 \\
(91.9 \%) \\
\text { Stage IV: } 3 \\
(8.1 \%)\end{array}$ & $\begin{array}{l}\text { Any type of treat- } \\
\text { ment }\end{array}$ \\
\hline Gupta (2018) & 24 & $16(67.7 \%)$ & $54(12.5)^{\mathrm{c}}$ & $\begin{array}{c}\text { ECOG 0: } 13 \\
(54.2 \%) \\
\text { ECOG 1: } 9 \\
(37.5 \%) \\
\text { ECOG 2: } 2 \\
(8.3 \%)\end{array}$ & & $\begin{array}{l}\text { Gastrointestinal: } \\
12(50.0 \%) \\
\text { Breast: } 4 \\
(16.7 \%) \\
\text { Lung: } 3(12.5 \%) \\
\text { Other: } 5 \\
(20.8 \%)\end{array}$ & & Chemotherapy \\
\hline $\begin{array}{l}\text { Broderick } \\
\text { (2019) }\end{array}$ & 42 & $21(50.0 \%)$ & $48.2[24-72]^{\mathrm{a}}$ & $\begin{array}{l}\text { ECOG 0: } 22 \\
(52.4 \%) \\
\text { ECOG 1: } 18 \\
(42.9 \%) \\
\text { ECOG 2: } 1 \\
(2.4 \%) \\
\text { Unknown: } 1 \\
(2.4 \%)\end{array}$ & & $\begin{array}{l}\text { Breast: } 17 \\
(40.5 \%) \\
\text { Testicular: } 10 \\
(23.8 \%) \\
\text { Head and neck: } \\
7(16.7 \%) \\
\text { Other: } 8 \\
(19.0 \%)\end{array}$ & $\begin{array}{l}\text { No evidence } \\
\text { disease: } 3 \\
(7.1 \%) \\
\text { Locally recur- } \\
\text { rent: } 14 \\
(33.3 \%) \\
\text { Distant metasta- } \\
\text { ses: } 21(50 \%)\end{array}$ & Chemotherapy \\
\hline Fujisawa (2019) & 41 & $21(51.2 \%)$ & $66.8(10.6)^{\mathrm{c}}$ & $\begin{array}{l}\text { ECOG 0: } 9 \\
(22.0 \%) \\
\text { ECOG 1: } 26 \\
(63.4 \%) \\
\text { ECOG 2: } 5 \\
(12.2 \%) \\
\text { ECOG 3: } 1 \\
(2.4 \%)\end{array}$ & & NSCLC & Stage IV & $\begin{array}{l}\text { Any type of treat- } \\
\text { ment }\end{array}$ \\
\hline Ohri (2019) & 50 & $20(40.0 \%)$ & $66[38-90]^{\mathrm{a}}$ & $\begin{array}{c}\text { ECOG 0: } 11 \\
(22.0 \%) \\
\text { ECOG 1: } 33 \\
(66.0 \%) \\
\text { ECOG 2: } 6 \\
(12.0 \%)\end{array}$ & & NSCLC & $\begin{array}{l}\text { Stage II: } 6 \\
(12.0 \%) \\
\text { Stage IIIA: } 24 \\
\quad(48.0 \%) \\
\text { Stage IIIB: } 16 \\
(32.0 \%) \\
\text { Stage IV: } 4 \\
(8.0 \%)\end{array}$ & $\begin{array}{l}\text { Chemoradio- } \\
\text { therapy }\end{array}$ \\
\hline
\end{tabular}

${ }^{\mathrm{a}}$ Mean [range]; ${ }^{\mathrm{b}}$ Median [range]; ${ }^{\mathrm{c}}$ Mean (SD); ${ }^{\mathrm{d}}$ Mean $\pm \mathrm{SD}$ [range]; $* 1$ patient recorded 2 primary cancer types; $* * 3$ patients on combination of treatments

ECOG-PS, Eastern Cooperative Oncology Group; PS, performance status; KPS, Karnofsky Performance Status; NSCLC, non-small cell lung carcinoma; $S C L C$, small cell lung carcinoma; $N H L$, non-Hodgkin lymphoma 


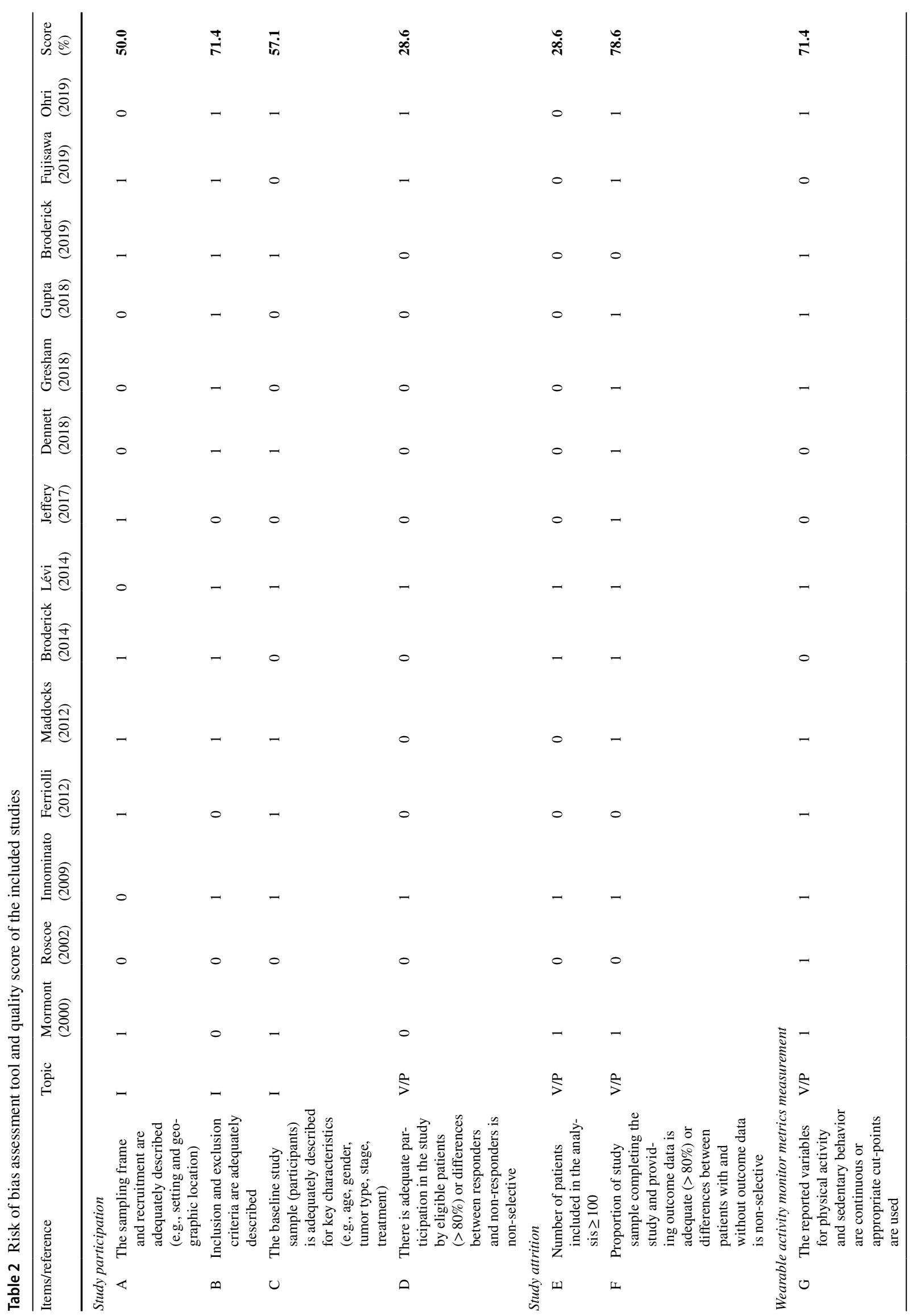




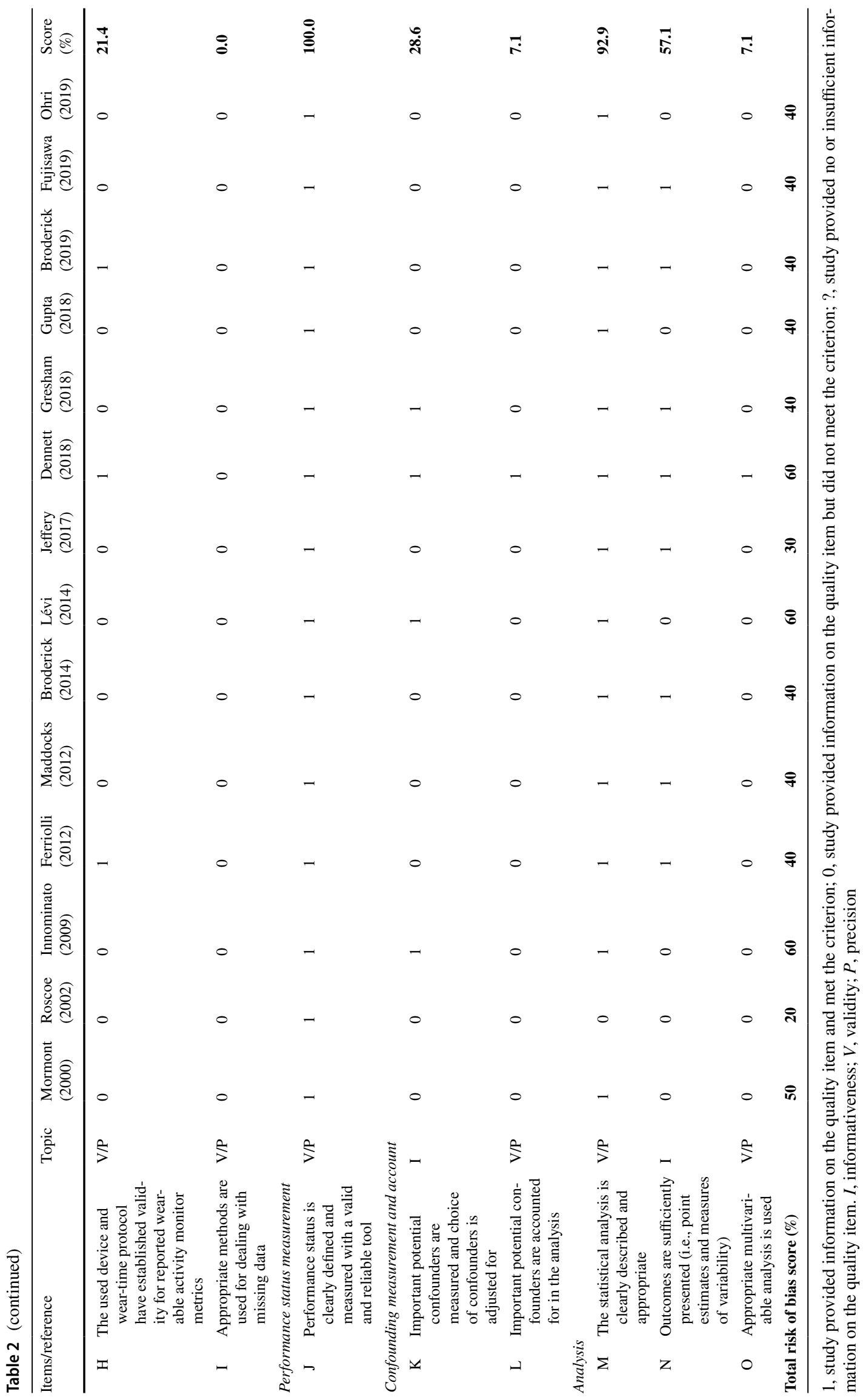




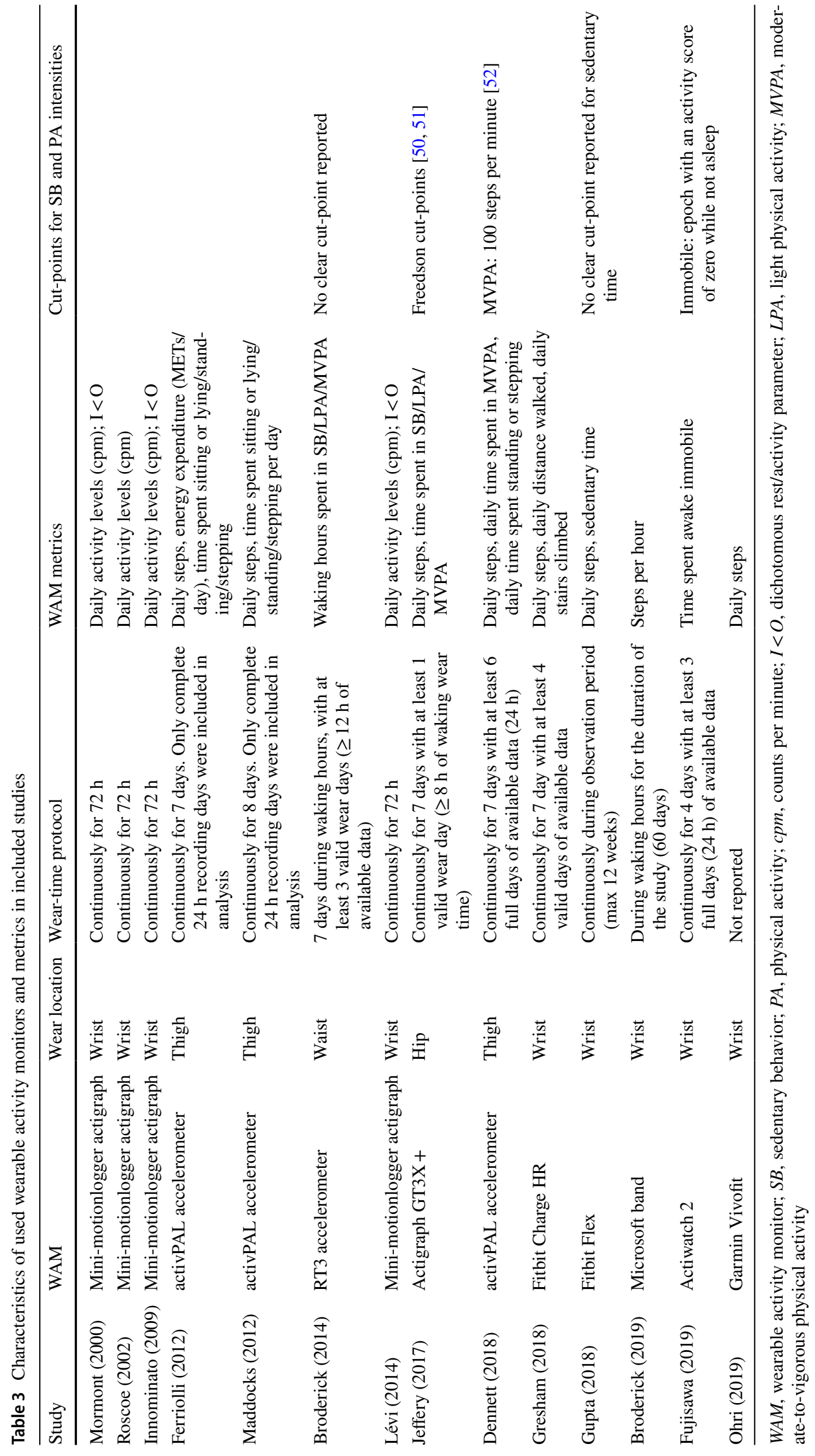




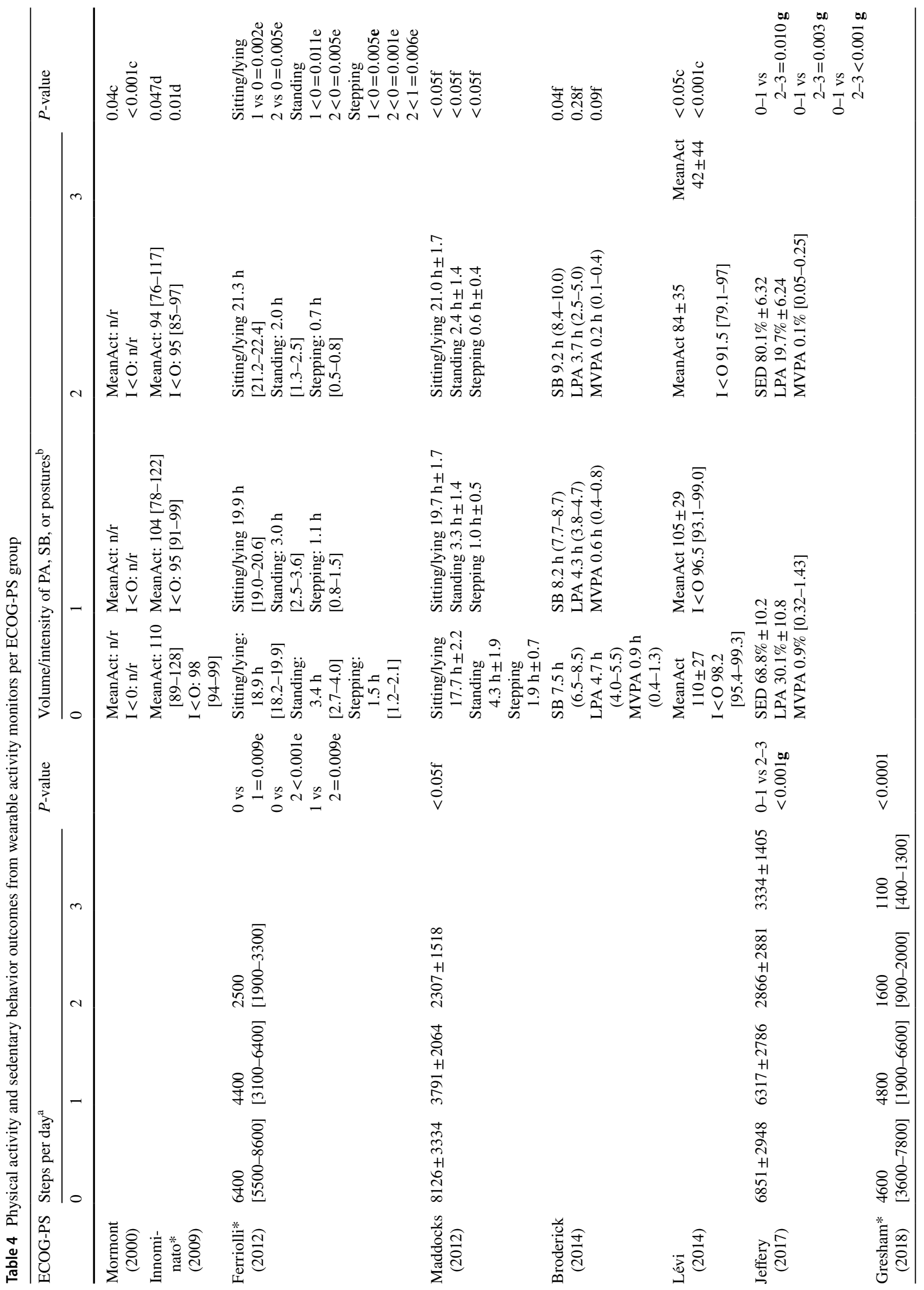




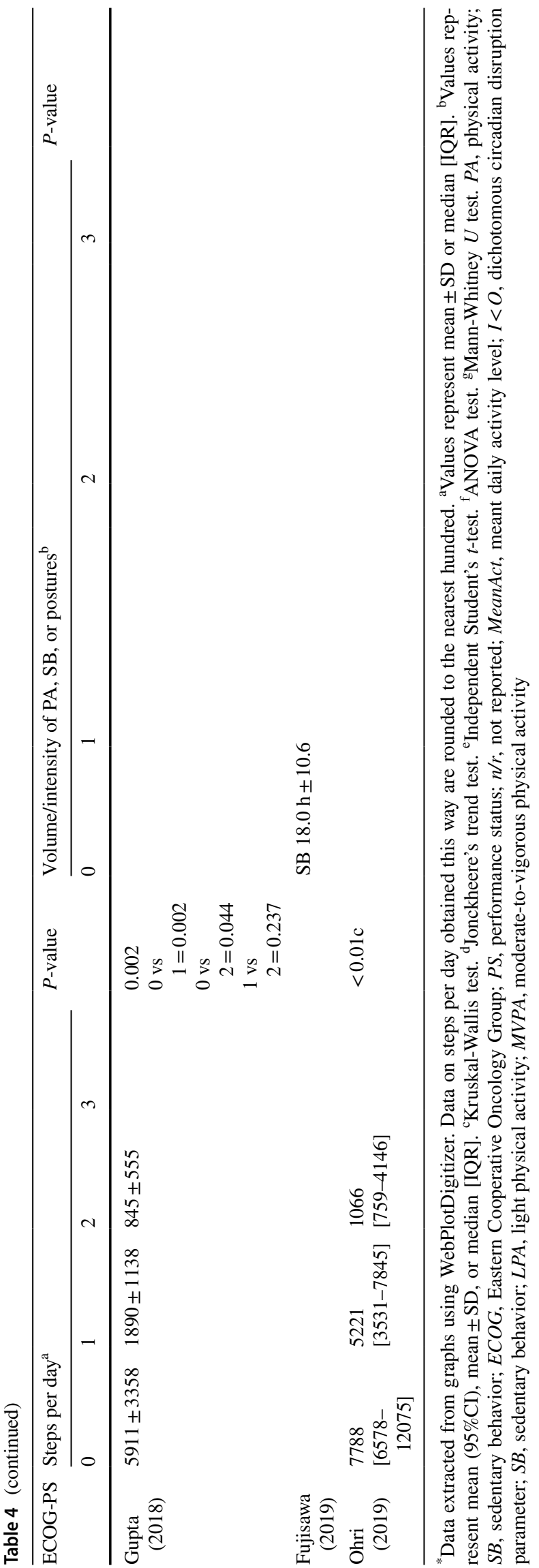

to patients with poor performance status [41]. Conversely, another study did not show significant differences regarding time spent in LPA and MVPA between groups based on performance status [42]. This study, however, reported that patients with better performance status spent significantly less time sedentary as compared to patients with worse performance status.

\section{Evidence synthesis for associations between wearable activity monitor metrics and performance status}

In total, we found 14 studies that could be included in the evidence synthesis. Results of evidence synthesis for the association between wearable activity monitor metrics and performance status are compiled in Table 5. We found moderate evidence for a moderate positive association between daily steps and performance status and moderate evidence for a weak positive association between activity counts and performance status. Moreover, we found moderate evidence for moderate positive associations between time spent standing/stepping and performance status and between the circadian rest-activity dichotomous index $\mathrm{I}<\mathrm{O}$ and performance status. Finally, we found moderate evidence for moderate negative associations between sedentary behavior (intensityor posture-based) and performance status.

\section{Discussion}

In this study, we reviewed the available evidence on the association between wearable activity monitor metrics and physician-assessed performance status. Evidence synthesis showed moderate evidence for weak-to-moderate positive associations between performance status and various wearable activity monitor metrics and a moderate negative association between performance status and sedentary behavior.

Different possible explanations can be provided for the absence of strong associations. First, these weak-tomoderate associations may suggest that wearable activity monitors and performance status scales assess different constructs of physical performance and cannot simply be interchanged. Wearable activity monitors objectively measure physical activity (levels) and can therefore be regarded as performance-based measurements that are independent of judgment. Performance status scales, on the other hand, are evaluation-based measurements that involve judgment using idiosyncratic criteria [5, 6]. Another possible explanation for the absence of strong associations can also be provided from a measurement perspective, with only few categories for physicians-assessed performance status. In the studies included in this review, only $12 \%$ of patients had poor performance status (ECOG-PS 2-3). The limited 
Table 5 Evidence synthesis for association between wearable activity monitor metrics and performance status

\begin{tabular}{|c|c|c|c|c|c|c|c|c|c|c|c|c|c|}
\hline \multirow[t]{2}{*}{ Study } & \multirow[t]{2}{*}{ RoB } & \multirow{2}{*}{$\begin{array}{ll}\text { Steps } \\
\text { Steps }\end{array}$} & \multicolumn{3}{|c|}{ Volume/intensity-based } & \multicolumn{3}{|c|}{ Volume/Posture-based } & \multicolumn{2}{|c|}{ Circadian rest/activity } & \multicolumn{3}{|c|}{ Other PA } \\
\hline & & & SB & LPA & MVPA & Sitting/lying & Standing & Stepping & MeanAct/cpm & $k 0$ & Distance & Stairs & METs \\
\hline $\begin{array}{l}\text { Mormont } \\
(2000)\end{array}$ & High & & & & & & & & $p=0.04$ & $p<0.001$ & & & \\
\hline $\begin{array}{l}\text { Roscoe } \\
(2002)\end{array}$ & High & & & & & & & & $\begin{array}{l}r=0.29 * \\
p<0.05\end{array}$ & & & & \\
\hline $\begin{array}{c}\text { Innominato } \\
\text { (2009) }\end{array}$ & High & $p<0.05$ & & & & & & & $p=0.047$ & $p=0.01$ & & & \\
\hline $\begin{array}{l}\text { Ferriolli } \\
(2012)\end{array}$ & High & $\begin{array}{l}r=0.545 \\
p=0.002\end{array}$ & & & & $\begin{array}{l}r=0.569 \\
p<0.001\end{array}$ & $\begin{array}{l}r=0.477 \\
p<0.001\end{array}$ & $\begin{array}{l}r=0.586 \\
p<0.001\end{array}$ & & & & & $\begin{array}{l}r=0.444 \\
p=0.016\end{array}$ \\
\hline $\begin{array}{c}\text { Maddocks } \\
\text { (2012) }\end{array}$ & High & $p<0.05$ & & & & $p<0.05$ & $p<0.05$ & $p<0.05$ & & & & & \\
\hline $\begin{array}{c}\text { Broderick } \\
\text { (2014) }\end{array}$ & High & & $p<0.05$ & $p=0.28$ & $p=0.09$ & & & & & & & & \\
\hline $\begin{array}{l}\text { Leví } \\
\text { (2014) }\end{array}$ & High & & & & & & & & $p<0.05$ & $p<0.05$ & & & \\
\hline $\begin{array}{l}\text { Dennett } \\
\text { (2017) }\end{array}$ & High & & & & $\begin{array}{l}r=0.393^{*} \\
p<0.05\end{array}$ & & & & & & & & \\
\hline $\begin{array}{l}\text { Jeffery } \\
\text { (2017) }\end{array}$ & High & $p<0.001$ & $p=0.01$ & $p=0.003$ & $p<0.001$ & & & & & & & & \\
\hline $\begin{array}{l}\text { Gresham } \\
\text { (2018) }\end{array}$ & High & $\begin{array}{l}r=0.63 \\
p<0.05\end{array}$ & & & & & & & & & $\begin{array}{l}r=0.61 \\
p<0.05\end{array}$ & $\begin{array}{l}r=0.57 \\
p>0.05\end{array}$ & \\
\hline $\begin{array}{l}\text { Gupta } \\
(2018)\end{array}$ & High & $p<0.001$ & & & & & & & & & & & \\
\hline $\begin{array}{c}\text { Broderick } \\
\text { (2019) }\end{array}$ & High & $\begin{array}{l}r=-0.28 \\
p>0.05\end{array}$ & & & & & & & & & & & \\
\hline $\begin{array}{l}\text { Fujisawa } \\
\text { (2019) }\end{array}$ & High & & $\begin{array}{l}r=0.365 \\
p<0.05\end{array}$ & & & & & & & & & & \\
\hline $\begin{array}{l}\text { Ohri } \\
(2019)\end{array}$ & High & $p=0.024$ & & & & & & & & & & & \\
\hline $\begin{array}{l}\text { Level of } \\
\text { evidence }\end{array}$ & & $\begin{array}{l}\text { moderate, } \\
\text { positive }\end{array}$ & $\begin{array}{c}\text { moderate, } \\
\text { negative }\end{array}$ & insufficient & insufficient & $\begin{array}{c}\text { moderate, } \\
\text { negative }\end{array}$ & $\begin{array}{c}\text { moderate, } \\
\text { positive }\end{array}$ & $\begin{array}{c}\text { moderate, } \\
\text { positive }\end{array}$ & $\begin{array}{c}\text { moderate, } \\
\text { positive }\end{array}$ & $\begin{array}{c}\text { moderate, } \\
\text { positive }\end{array}$ & insufficient & insufficient & insufficient \\
\hline \multicolumn{14}{|c|}{$\begin{array}{l}\text { Associations shown with ECOG PS, unless stated otherwise. *association with KPS. ECOG PS, Easter Cooperative Oncology Group Performance Status; } K P S, \text { Karnofsky Performance Status. RoB, risk of bias; } P A \text {, } \\
\text { physical activity; } S B \text {, sedentary behavior; } L P A \text {, light physical activity; } M V P A \text {, moderate-to-vigorous physical activity; MeanAct, mean activity levels; } c p m \text {, counts per minute; } M E T \text {, metabolic equivalent of task }\end{array}$} \\
\hline & & \multicolumn{4}{|c|}{ Positive association } & \multicolumn{2}{|c|}{$\begin{array}{l}\text { Moderate grade evidence } \\
\text { for positive association }\end{array}$} & & & & & & \\
\hline & & \multicolumn{2}{|c|}{ Negative association } & & & \multicolumn{2}{|c|}{$\begin{array}{l}\text { Moderate grade evidence } \\
\text { for negative association }\end{array}$} & & & & & & \\
\hline & & \multicolumn{2}{|c|}{ Non-significant association } & & & \multicolumn{2}{|c|}{$\begin{array}{l}\text { Insufficient evidence for } \\
\text { association }\end{array}$} & & & & & & \\
\hline
\end{tabular}

Associations shown with ECOG PS, unless stated otherwise. *association with KPS. ECOG PS, Easter Cooperative Oncology Group Performance Status; $K P S$, Karnofsky Performance Status. RoB, Risk of bias; $P A$, physical activity; $S B$, sedentary behavior; $L P A$, light physical activity; $M V P A$, moderate-to-vigorous physical activity; MeanAct, mean activity levels; $c p m$, counts per minute; MET, metabolic equivalent of task

variation in scoring could have contributed to the absent, or weak-to-moderate associations. Furthermore, substantial heterogeneity across studies in terms of devices used, weartime protocols, study population, and methodology could be a potential source for the absence of strong associations between wearable activity monitor metrics and performance status.

More detailed objectively and passively gathered activity data from wearable activity monitors might be of added value in clinical practice. Wearable activity monitorassessed physical activity/sedentary behavior might serve as a dynamic and objective supplement measurement of patients' performance status as assessed by the physician and, as such, might prove to be of added value in clinical decision making and evaluation of treatment options in oncology. This hypothesis is substantiated by observations that more daily steps are associated with lower risk of hospitalization during cancer treatments [33, 43], longer survival [33, 41, 44], and lower chance of serious adverse events [33, 45]. Interestingly, Fujisawa et al. demonstrated that among patients with good performance status (ECOGPS 0-1), ECOG-PS was not predictive for survival, while sedentary behavior was a significant predictor for 6-month survival [36]. Moreover, Jeffery et al. reported that patients with a survival longer than 3 months spent significantly less time sedentary as compared to those who survived less than 3 months [41]. Together, these results suggest potential value of objective sedentary behavior measurement in predicting survival outcomes, especially in patients with good PS. In this way, wearable activity monitors might assist physicians in clinical decision making, like determining whether a patient is fit for treatment.

Most currently available wearable activity monitors (e.g., Fitbit Charge HR) are multisensory devices that have a builtin 3D-accelerometer as well as other sensors that measure, for example, heart rate. In the era of advancing artificial intelligence and machine learning, it is very conceivable that data input from combinations of wearable activity monitor sensors and data patterns over time might prove to be superior in assessing performance status and predicting outcomes for patients with cancer than physician-assessed performance status.

Recently, various pilot studies have demonstrated the feasibility of using wearable activity monitors in the context of an ambulatory monitoring platform that longitudinally assesses treatment-related adverse events, unplanned healthcare encounters, and survival in patients with cancer [33, 46, 47]. Results from these studies suggest potential for wearable activity monitors in early detection of adverse event and unplanned healthcare encounters. The application 
of wearable activity monitors in this context has a lot of potential to be clinically impactful and improve cancer care. Future research should focus on proving the efficacy of wearable activity monitors as a part of ambulatory monitoring platforms.

An important finding of our systematic review is the high heterogeneity between included studies regarding study population, devices used, wear-time protocols, definitions and cut-points used for different physical activity metrics, and reporting of outcomes, thereby hindering adequate comparison of results on the association between physical activity metrics and performance status and complicating best evidence synthesis. A second limitation of the studies included in this review is the high risk of bias scores. Major factors contributing to the high risk of bias scores were unvalidated methods regarding physical activity measurements, low sample sizes, and the lack of multivariable analyses to adjust for relevant confounders. It should be noted that the majority of these studies investigated the association between wearable activity monitor metrics and performance status as secondary or exploratory analysis, which may have contributed to the high risk of bias scores. Consequently, it is currently also unclear whether the association between wearable activity monitor metrics and performance status varies by cancer type or stage. With regard to the physical activity measurements, none of the studies adequately reported on the handling of missing physical activity data. Different studies have emphasized the need for missing accelerometer data imputation and suggested statistical methods of handling missing data [48, 49]. Moreover, the majority of studies used devices, weartime protocols, or cutoff points that have not adequately been validated in comparable populations. Taken together, results should be interpreted with caution and emphasize that standardization of wearable activity monitor-measured physical activity and sedentary behavior methodology is warranted to decrease risk of bias in future studies on the subject.

Strengths of this systematic review include the in-depth risk of bias assessment that was adjusted specifically for studies using a wearable activity monitor for physical activity and sedentary behavior measurements and the subsequent best evidence synthesis. However, most of the included studies were not designed to investigate the association between wearable activity monitor metrics and performance status, complicating risk of bias assessment and evidence synthesis. More than half of the included studies were designed to investigate physical activity levels in specific cancer populations, study the feasibility of wearable activity monitors, or explore associations between other wearable activity monitor metrics, like circadian rest-activity rhythm parameters, and various outcomes. Therefore, the association between wearable activity monitor metrics and performance status was often analyzed in a secondary or exploratory analysis resulting in suboptimal presenting of results. Moreover, results may be prone to reporting bias as non-significant associations are less likely to be reported, resulting in an overestimation of the associations between wearable activity monitor metrics and performance status.

In conclusion, we found moderate evidence for a positive weak-to-moderate association between various physical activity metrics and performance status and for an inverse moderate association between sedentary behavior and performance status. The strength of the associations should be interpreted with caution given the aforementioned limitations of the available evidence. Nevertheless, our results suggest that objectively measured physical activity may serve as a dynamic and objective supplement measurement of a patient's functional performance status and may be of added value in clinical decision making and evaluation of treatment options in oncology. Next steps include to study the association between wearable activity monitor metrics and clinical outcomes and directly compare the predictive value of objectively measured physical activity with performance status for relevant clinical outcomes. Finally, consensus is warranted on the methodology of objective physical activity measurement and efforts should be made to validate the different methods (i.e., device, parameters, wear-time protocols) in relevant patient populations.

Supplementary Information The online version contains supplementary material available at https://doi.org/10.1007/s00520-021-06234-5.

Author contribution M.K, L.B, H.W.M.v.L, and M.G.H.v.O contributed to study concepts. M.K, E.P, L.B, C.K, J.W.W H.W.M.v.L, and M.G.H.v.O contributed to study design. M.K, E.P, and B.B contributed to data acquisition. M.K, E.P, B.B, C.K, L.B, H.W.M.v.L, and M.G.H.v.O contributed to data analysis and interpretation. M.K, E.P, C.K, L.B, H.W.M.v.L, and M.G.H.v.O contributed to manuscript preparation. M.K, E.P, L.B, C.K, B.B, J.W.W, H.W.M.v.L, and M.G.H.v.O contributed to manuscript editing. L.B, J.W.W, H.W.M.v.L, and M.G.H.v.O contributed to manuscript review. All authors gave the final approval for submission of the manuscript.

Data availability Data are available on request from the authors.

Code availability Not applicable.

\section{Declarations}

Ethics approval Not applicable.

Consent to participate Not applicable.

Consent for publication Not applicable.

Conflict of interest M.G.H. van Oijen has received unrestricted research grants from BMS, Lilly, Merck Serono, Nordic, and Servier, outside the submitted work. H.W.M. van Laarhoven has served as a consultant for BMS, Celgene, Lilly, and Nordic and has received unrestricted research grants from Bayern, BMS, Celgene, Lilly, Merck Serono, MSD, Nordic, Philips, and Roche, outside the submitted work. J.W. Wilmink has served as a consultant for Celgene, Servier, and 
Shire and has received unrestricted research grants from Amgen, AstraZeneca, Celgene, Halozyme, Merck, Novartis, Pfizer, Roche, and Servier, outside the submitted work. The other authors have nothing to declare.

Open Access This article is licensed under a Creative Commons Attribution 4.0 International License, which permits use, sharing, adaptation, distribution and reproduction in any medium or format, as long as you give appropriate credit to the original author(s) and the source, provide a link to the Creative Commons licence, and indicate if changes were made. The images or other third party material in this article are included in the article's Creative Commons licence, unless indicated otherwise in a credit line to the material. If material is not included in the article's Creative Commons licence and your intended use is not permitted by statutory regulation or exceeds the permitted use, you will need to obtain permission directly from the copyright holder. To view a copy of this licence, visit http://creativecommons.org/licenses/by/4.0/.

\section{References}

1. Sargent DJ, Köhne CH, Sanoff HK et al (2009) Pooled safety and efficacy analysis examining the effect of performance status on outcomes in nine first-line treatment trials using individual data from patients with metastatic colorectal cancer. J Clin Oncol 27:1948-1955

2. Mol L, Ottevanger PB, Koopman M, Punt CJA (2016) The prognostic value of WHO performance status in relation to quality of life in advanced colorectal cancer patients. Eur J Cancer 66:138-143

3. ter Veer E, van Kleef JJ, Schokker S et al (2018) Prognostic and predictive factors for overall survival in metastatic oesophagogastric cancer: a systematic review and meta-analysis. Eur J Cancer 103:214-226

4. ter Veer E, van Rijssen LB, Besselink MG et al (2018) Consensus statement on mandatory measurements in pancreatic cancer trials (COMM-PACT) for systemic treatment of unresectable disease. Lancet Oncol 19:e151-e160

5. Karnofsky D, Burchenal J (1949) In CM MacLeod (Ed.), Evaluation of chemotherapeutic agents. undefined

6. Oken MM, Creech RH, Tormey DC et al (1982) Toxicity and response criteria of the Eastern Cooperative Oncology Group. Am J Clin Oncol 5:649-656

7. Kelly CM, Shahrokni A (2016) Moving beyond Karnofsky and ECOG performance status assessments with new technologies. J Oncol 2016:6186543

8. Myers J, Gardiner K, Harris K et al (2010) Evaluating correlation and interrater reliability for four performance scales in the palliative care setting. J Pain Symptom Manag 39:250-258

9. Blagden SP, Charman SC, Sharples LD et al (2003) Performance status score: do patients and their oncologists agree? Brit J Cancer 89:1022

10. Taylor AE, Olver IN, Sivanthan T et al (1999) Observer error in grading performance status in cancer patients. Support Care Cancer 7:332-335

11. Chow R, Chiu N, Bruera E et al (2016) Inter-rater reliability in performance status assessment among health care professionals: a systematic review. Ann Palliat Medicine 5:83-92

12. Ando M, Ando Y, Hasegawa $Y$ et al (2001) Prognostic value of performance status assessed by patients themselves, nurses, and oncologists in advanced non-small cell lung cancer. Brit J Cancer $85: 1634-1639$
13. Sallis JF, Saelens BE (2000) Assessment of physical activity by self-report: status, limitations, and future directions. Res Q Exercise Sport 71:1-14

14. Scott JM, Stene G, Edvardsen E, Jones LW (2020) Performance status in cancer: not broken, but time for an upgrade? J Clin Oncol 38:2824-2829

15. ter Veer E, Mohammad NH, van Valkenhoef G et al (2016) The efficacy and safety of first-line chemotherapy in advanced esophagogastric cancer: a network meta-analysis. J Natl Cancer I 108:djw166

16. Mohammad NH, ter Veer E, Ngai L et al (2015) Optimal firstline chemotherapeutic treatment in patients with locally advanced or metastatic esophagogastric carcinoma: triplet versus doublet chemotherapy: a systematic literature review and meta-analysis. Cancer Metastasis Rev 34:429-441

17. Moher D, Liberati A, Tetzlaff J et al (2010) Preferred reporting items for systematic reviews and meta-analyses: the PRISMA statement. Int J Surg 8:336-341

18. Burda BU, O'Connor EA, Webber EM et al (2017) Estimating data from figures with a web-based program: considerations for a systematic review. Res Synth Methods 8:258-262

19. Drevon D, Fursa SR, Malcolm AL (2017) Intercoder reliability and validity of WebPlotDigitizer in extracting graphed data. Behav Modif 41:323-339

20. Moeyaert M, Maggin D, Verkuilen J (2016) Reliability, validity, and usability of data extraction programs for single-case research designs. Behav Modif 40:874-900

21. Evans JD (1996) Straightforward statistics for the behavioral sciences. Thomson Brooks/Cole Publishing Co., Pacific Grove

22. Hayden JA, Côté P, Bombardier C (2006) Evaluation of the quality of prognosis studies in systematic reviews. Ann Intern Med $144: 427$

23. Kampshoff CS, Jansen F, van Mechelen W et al (2014) Determinants of exercise adherence and maintenance among cancer survivors: a systematic review. Int J Behav Nutr Phy 11:80

24. Füzéki E, Engeroff T, Banzer W (2017) Health benefits of lightintensity physical activity: a systematic review of accelerometer data of the National Health and Nutrition Examination Survey (NHANES). Sports Med 47:1769-1793

25. Chinapaw MJM, Proper KI, Brug J et al (2011) Relationship between young peoples' sedentary behaviour and biomedical health indicators: a systematic review of prospective studies. Obes Rev 12:e621-e632

26. Cliff DP, Hesketh KD, Vella SA et al (2016) Objectively measured sedentary behaviour and health and development in children and adolescents: systematic review and meta-analysis. Obes Rev $17: 330-344$

27. Uijtdewilligen L, Nauta J, Singh AS et al (2011) Determinants of physical activity and sedentary behaviour in young people: a review and quality synthesis of prospective studies. Brit J Sport Med 45:896

28. van Nieuwenhuizen AJ, Buffart LM, Brug J et al (2015) The association between health related quality of life and survival in patients with head and neck cancer: a systematic review. Oral Oncol 51:1-11

29. Roscoe JA, Morrow GR, Hickok JT et al (2002) Temporal interrelationships among fatigue, circadian rhythm and depression in breast cancer patients undergoing chemotherapy treatment. Support Care Cancer 10:329-336

30. Mormont MC, Waterhouse J, Bleuzen P et al (2000) Marked 24-h rest/activity rhythms are associated with better quality of life, better response, and longer survival in patients with metastatic colorectal cancer and good performance status. Clin Cancer Res 6:3038-3045 
31. Innominato PF, Focan C, Gorlia T et al (2009) Circadian rhythm in rest and activity: a biological correlate of quality of life and a predictor of survival in patients with metastatic colorectal cancer. Cancer Res 69:4700-4707

32. Lévi F, Dugué P-A, Innominato P et al (2014) Wrist actimetry circadian rhythm as a robust predictor of colorectal cancer patients survival. Chronobiol Int 31:891-900

33. Gresham G, Hendifar AE, Spiegel B et al (2018) Wearable activity monitors to assess performance status and predict clinical outcomes in advanced cancer patients. Npj Digital Medicine 1:27. https://doi.org/10.1038/s41746-018-0032-6

34. Gupta A, Stewart T, Bhulani N et al (2018) Feasibility of wearable physical activity monitors in patients with cancer. Jco Clin Cancer Informatics 2018:1-10

35. Broderick JE, May M, Schwartz JE et al (2019) Patient reported outcomes can improve performance status assessment: a pilot study. J Patient-reported Outcomes 3:41

36. Fujisawa D, Temel JS, Greer JA et al (2019) Actigraphy as an assessment of performance status in patients with advanced lung cancer. Palliat Supportive Care 17:574-578

37. Ohri N, Halmos B, Bodner WR et al (2019) Daily step counts: a new prognostic factor in locally advanced non-small cell lung cancer? Int J Radiat Oncol Biology Phys 105:745-751

38. Ferriolli E, Skipworth RJE, Hendry P et al (2012) Physical activity monitoring: a responsive and meaningful patient-centered outcome for surgery, chemotherapy, or radiotherapy? J Pain Symptom Manag 43:1025-1035

39. Maddocks M, Wilcock A (2012) Exploring physical activity level in patients with thoracic cancer: implications for use as an outcome measure. Support Care Cancer 20:1113-1116

40. Dennett AM, Peiris CL, Shields N et al (2018) Cancer survivors awaiting rehabilitation rarely meet recommended physical activity levels. Rehabilitation Oncol 36:214-222

41. Jeffery E, Lee YCG, McVeigh J et al (2017) Feasibility of objectively measured physical activity and sedentary behavior in patients with malignant pleural effusion. Support Care Cancer 25:3133-3141

42. Broderick JM, Hussey J, Kennedy MJ, Donnell DMO (2014) Patients over 65 years are assigned lower ECOG PS scores than younger patients, although objectively measured physical activity is no different. J Geriatr Oncol 5:49-56

43. Ohri N, Kabarriti R, Bodner WR et al (2017) Continuous activity monitoring during concurrent chemoradiotherapy. Int J Radiat Oncol Biology Phys 97:1061-1065

44. Dorion V, Lambert L, Frazzi A et al (2017) A pilot study in the use of activity trackers for assessing response to palliative radiotherapy. Cureus 9:e1871

45. Soto-Perez-De-Celis E, Kim H, Rojo-Castillo MP et al (2018) A pilot study of an accelerometer-equipped smartphone to monitor older adults with cancer receiving chemotherapy in Mexico. $\mathrm{J}$ Geriatr Oncol 9:145-151

46. Nilanon T, Nocera LP, Martin AS et al (2020) Use of wearable activity tracker in patients with cancer undergoing chemotherapy: toward evaluating risk of unplanned health care encounters. Jco Clin Cancer Informatics 4:839-853

47. Wright AA, Raman N, Staples P et al (2018) The HOPE pilot study: harnessing patient-reported outcomes and biometric data to enhance cancer care. Jco Clin Cancer Informatics 2018:1-12

48. Lee PH (2013) Data imputation for accelerometer-measured physical activity: the combined approach. Am J Clin Nutrition 97:965-971

49. Stephens S, Beyene J, Tremblay MS et al (2018) Strategies for dealing with missing accelerometer data. Rheum Dis Clin N Am 44:317-326

50. Freedson PS, Melanson E, Sirard J (1998) Calibration of the Computer Science and Applications. Inc accelerometer Medicine Sci Sports Exerc 30:777-781

51. Matthews CE, Chen KY, Freedson PS et al (2008) Amount of time spent in sedentary behaviors in the United States, 2003-2004. Am J Epidemiol 167:875-881

52. Tudor-Locke C, Craig CL, Aoyagi Y et al (2011) How many steps/ day are enough? For older adults and special populations. Int J Behav Nutr Phy 8:80

Publisher's note Springer Nature remains neutral with regard to jurisdictional claims in published maps and institutional affiliations. 